\title{
The CXCL12/CXCR4 autocrine loop increases the metastatic potential of non-small cell lung cancer in vitro
}

\author{
XIAOFENG DAI, ZHIFU MAO, JIE HUANG, SONGPING XIE and HAO ZHANG \\ Department of Cardiothoracic Surgery, Renmin Hospital of Wuhan University, Wuhan, Hubei 430060, P.R. China
}

Received July 3, 2012; Accepted September 25, 2012

DOI: $10.3892 / \mathrm{ol} .2012 .960$

\begin{abstract}
The CXCL12/CXCR4 endocrine axis has been demonstrated to play a pivotal role in organ-specific metastasis of many different types of tumors, but the precise role of the CXCL12/CXCR4 autocrine loop remains poorly understood. In this study, we constructed a functional CXCL12/CXCR4 autocrine loop in A549 cells using a gene transfection technique to evaluate its effect on the metastasis of non-small cell lung cancer (NSCLC). Our results demonstrated that the CXCL12/CXCR4 autocrine loop significantly promoted the motility, proliferation and invasiveness of the A549 cells, suggesting a key role of the CXCL12/CXCR4 autocrine loop in NSCLC metastasis. In addition, these findings suggest that targeted therapies directed against CXCR4 should consider the CXCL12 expression status of the NSCLC to be treated, since tumors with autocrine overexpression of CXCL12 may be more suitable for the application of chemokine-based anticancer therapies.
\end{abstract}

\section{Introduction}

Chemokines are a large family of small (7-15 kDa), structurally related heparin-binding proteins that bind to and activate a family of chemokine receptors. More than 50 chemokines have been identified and they are classified into 4 families (CXC, CX3C, CC and C) based on the position of the first two conserved cysteine residues (1). Chemokine receptors are 7-transmembrane $\mathrm{G}$ protein-coupled receptors. Most are promiscuous and can bind with high affinity to multiple chemokine ligands (CXCR, CX3CR, CCR and XCR). At present, 20 chemokine receptors have been identified.

In normal physiology, chemokines are involved in proinflammatory and non-inflammatory cell homing by binding to their cognate receptors (2). However, increasing evidence

Correspondence to: Professor Zhifu Mao, Department of Cardiothoracic Surgery, Renmin Hospital of Wuhan University, 238 Jiefang Road, Wuhan, Hubei 430060, P.R. China

E-mail: mao.zhifu@yahoo.cn

Key words: CXCL12/CXCR4 autocrine loop, chemokine, metastasis, non-small cell lung cancer implicates these small cytokine-like proteins and their receptors in tumor biology (3-5). CXCL12 and its cognate receptor CXCR4 have been shown to regulate site-specific distant metastasis of many cancer types (6-10). These studies demonstrated that tumor cells express a high level of CXCR4 and that tumor metastasis target tissues (lung, liver and bone) express high levels of the ligand CXCL12, allowing tumor cells to directionally migrate to target organs via a CXCL12-CXCR4 chemotactic gradient. These studies have led to the current CXCL12/CXCR4 'endocrine axis' model; CXCR4 expression by metastatic cells enables those cells to navigate towards organs abundantly expressing CXCL12. Currently, despite compelling evidence for the pro-metastatic function of the CXCL12/CXCR4 endocrine axis, little attention has been devoted to the precise role of the CXCL12/CXCR4 autocrine loop in tumor metastasis.

A previous study reported a pro-metastatic effect of the CXCL12/CXCR4 autocrine loop when CXCL12 was transfected into the mammary carcinoma cell line MDA-MB-231 (11). This study also demonstrated that CXCL12 expression was inversely correlated with disease-free and overall survival in breast cancer patients. In another study, increased proliferation was in contrast to reduced metastasis in vivo, which has been demonstrated as responsible for forced CXCL12-expressing MDA-MB-231 cells (12). These contradictory results suggest that the function of the CXCL12/CXCR4 autocrine loop in tumor growth and metastasis requires further elucidation.

Matrix metalloproteinases (MMPs), which are multidomain zinc-dependent endopeptidases, are pivotal in cancer invasion and metastasis $(13,14)$. Among these MMPs, MMP-2 and MMP-9 have been of particular interest due to their pathogenic roles in non-small cell lung cancer (NSCLC) (15-17). Degradation of ECM and basement membranes by MMP-2 and/or MMP-9 is required for tumor cell invasion and metastasis $(16,18,19)$. The correlation between the CXCL12/CXCR4 autocrine loop and the expression of MMP-2 and MMP-9 remains unknown.

We performed this study to investigate the previously unknown role of the CXCL12/CXCR4 autocrine loop in cell growth and distant metastasis of NSCLC. This was achieved by using a gene transfection technique. Human NSCLC cell line A549, which does not express endogenous CXCL12, was transfected with pIRES2-ZsGreen1-CXCL12 or the control vector pIRES2-ZsGreen1 to establish stable CXCL12 (A549-CXCL12) and control vector (A549-ZsGreen1) 
transfectants. In the stable CXCL12-expressing cell line (A549-CXCL12), a functional CXCL12/CXCR4 autocrine loop was constructed. The contribution of the CXCL12/ CXCR4 autocrine loop signaling pathway to the migration, proliferation and invasiveness of A549-CXCL12 cells was evaluated and compared with A549-ZsGreen1 and wild-type A549 cells. We hypothesized that the CXCL12/CXCR4 autocrine loop may stimulate production of MMP-2 and MMP-9. Therefore, we also investigated whether the CXCL12/CXCR4 autocrine loop affected MMP-2 and MMP-9 expression in NSCLC cells.

\section{Materials and methods}

Materials. Anti-mouse and anti-rabbit IgG-conjugated horseradish peroxidase and rabbit polyclonal antibodies specific for extracellular signal-regulated kinase (ERK), phosphorylated (p-) ERK, MMP-9 and MMP-2 were purchased from Santa Cruz Biotechnology (Santa Cruz, CA, USA). Rabbit polyclonal antibodies specific for GAPDH, CXCR4 and CXCL12 were purchased from R\&D Systems (Minneapolis, MN, USA). Lipofectamine ${ }^{\circledR} 2000$ reagent was purchased from Invitrogen Ltd. (Paisley, UK). The human lung cancer cell lines A549 and H1975 were purchased from American Type Culture Collection (Rockville, MD, USA), and 95C and 95D cell lines were obtained from China Center for Type Culture Collection (Wuhan, China). Human full-length CXCL12 was purchased from Biofavor Ltd. (Wuhan, China) and the pIRES2-ZsGreen1 vector was purchased from Takara Biotechnology (Dalian, China). PCR primers were designed using Beacon Designer (Palo Alto, CA, USA) and synthesized by Yingjun Biotechnology (Shanghai, China). Molecular biology-grade agarose was obtained from Invitrogen Ltd. The RNA extraction and reverse transcription kits were obtained from AbGene Ltd. (Epsom, UK). Matrigel was purchased from BD Biosciences (San Jose, CA, USA). A Transwell plate equipped with a porous insert (pore size, $8 \mu \mathrm{m}$ ) was obtained from Becton Dickinson Labware (Franklin Lakes, NJ, USA).

Cell culture. Human NSCLC cell lines A549, H1975, 95C and 95D were maintained in RPMI-1640 medium supplemented with $10 \%$ fetal calf serum, $100 \mathrm{U} / \mathrm{ml}$ penicillin and $100 \mu \mathrm{g} / \mathrm{ml}$ streptomycin in a humid atmosphere of $5 \% \mathrm{CO}_{2}$ and $95 \%$ air, at $37^{\circ} \mathrm{C}$. This study was approved by the Ethics Committee of Renmin Hospital of Wuhan University, Wuhan, China.

Stable transfection. The human full-length CXCL12 cDNA fragment was ligated to the cloning site of pIRES2ZsGreen1, followed by transformation using One-Shot ${ }^{\circledR}$ E.coli (Invitrogen Ltd.), verification and amplification. A549 cells were transfected with either purified or control plasmid using Lipofectamine 2000 reagent (according to the manufacturer's instructions) followed by selection with G418. Stable CXCL12 (A549-CXCL12) and control plasmid (A549-ZsGreen1) transfectants were subsequently established and verified.

Western blot analyses and reverse transcription-PCR (RT-PCR) analyses. Proteins in the total cell lysates were resolved by SDS-PAGE and electrotransferred to a polyvinylidene difluoride membrane (Millipore, Bedford, MA, USA).
After the blot was blocked in a solution of $4 \%$ bovine serum albumin, membrane-bound proteins were probed overnight with primary antibodies against GAPDH, CXCL12, CXCR4, MMP-2, MMP-9 or p-ERK. They were then incubated with horseradish peroxidase-conjugated secondary antibodies for $1 \mathrm{~h}$. Antibody-bound protein bands were detected with enhanced chemiluminescence reagents and photographed with Kodak X-OMAT LS film (Eastman Kodak, Rochester, NY, USA). Quantitative data were obtained using a computing densitometer and ImageQuant software (Molecular Dynamics, Sunnyvale, CA, USA).

For RT-PCR analysis, RNA was extracted from total cell lysates using a TRIzol kit (MDBio, Piscataway, NJ, USA). The RNA concentration was determined using an ultraviolet spectrophotometer. The reverse transcription reaction was performed using $2 \mu \mathrm{g}$ total RNA that was reverse-transcribed into cDNA using oligo(dT) primer, then amplified for 30 cycles using 2 oligodeoxynucleotide primers: $\beta$-actin sense, 5'-CACGATGGAGGGGCCGGACTCATC-3' and anti-sense, 5'-TAAAGACCTCTATGCCAACACAGT-3'; CXCL12 sense, 5'-GTCAGCCTGAGCTACAGATGC-3' and anti-sense, 5'-CTTTAGCTTCGGGTCAATGC-3'; CXCR4 sense, 5'-CCGTGGCAAACTGGTACTTT-3' and anti-sense, 5'-GACGCCAACATAGACCACCT-3'; MMP-2 sense, 5'-GTGCTGAAGGACACACTAAAGAAGA-3' and anti-sense, 5'-TTGCCATCCTTCTCAAAGTTGTAGC-3'; or MMP-9 sense, 5'-CACTGTCCACCCCTCAGAGC-3' and anti-sense, 5'-GCCACTTGTCGGCGATAAGC-3'. PCR was carried out as follows: $94^{\circ} \mathrm{C}$ for $4 \mathrm{~min}$, followed by 30 cycles of $94^{\circ} \mathrm{C}$ for $30 \mathrm{sec}, 52^{\circ} \mathrm{C}$ for $30 \mathrm{sec}, 72^{\circ} \mathrm{C}$ for $25 \mathrm{sec}$ and a final extension for $4 \mathrm{~min}$ at $72^{\circ} \mathrm{C}$. The products were visualized on $2 \%$ agarose gel after staining with ethidium bromide.

Wound assay. Cells were grown to confluence in 6-well plates and starved with serum-free RPMI-1640 medium for $24 \mathrm{~h}$. An injury line was created with a pipette tip in the center of the dishes. Following rinsing with phosphate-buffered saline (PBS), cells were allowed to migrate for $12 \mathrm{~h}$ and then photographed (x100). Each clone was plated and wounded in triplicate and each experiment was repeated at least 3 times.

Invasion assay. The invasion assay was performed using Transwells with filter inserts (pore size, $8 \mu \mathrm{m}$ ) coated with $50 \mu$ l Matrigel diluted 1:3. Approximately $2.5 \times 10^{4}$ cells in $100 \mu 1$ serum-free RPMI-1640 medium were placed in the upper chamber and $1 \mathrm{ml}$ of the same medium was placed in the lower chamber. Following $18 \mathrm{~h}$ incubation at $37^{\circ} \mathrm{C}$ in $5 \% \mathrm{CO}_{2}$, non-invading cells were removed from the upper surface of the filter. Cells that had migrated through the filter were fixed with $4 \%$ paraformaldehyde (Sigma, St. Louis, MO, USA), stained with crystal violet for Matrigel and counted under a microscope (x400). Each clone was plated in triplicate in each experiment, and each experiment performed in triplicate.

MTT assay. Briefly, $1 \times 10^{4}$ cells were seeded onto 96-well plates and allowed to adhere for $8 \mathrm{~h}$. Cell proliferation was assessed at various time points by adding $10 \mu \mathrm{l}$ filter-sterilized MTT $(5 \mathrm{mg} / \mathrm{ml}$ in PBS) to a single row of 6 wells. Following $4 \mathrm{~h}$ incubation with MTT, the media was removed and the 
blue formazan crystals trapped in the cells were dissolved in $100 \mu \mathrm{l}$ sterile DMSO, by incubating at $37^{\circ} \mathrm{C}$ for $30 \mathrm{~min}$. Absorbance at $568 \mathrm{~nm}$ was measured in each well with a plate reader. The growth curve was constructed by plotting absorbance against time.

Statistics. Statistical differences between the means of the different groups were evaluated with Prism 5.01 (GraphPad Software Inc., La Jolla, CA, USA) using one-way ANOVA. $\mathrm{P}<0.05$ was considered to indicate a statistically significant difference.

\section{Results}

Expression pattern of CXCL12 and CXCR4 in NSCLC cell lines. Several studies have demonstrated CXCR4 expression in NSCLC cell lines, but its ligand CXCL12 has not been observed $(10,15,20)$. We examined mRNA expression of CXCL12 and CXCR4 in a panel of human NSCLC cell lines, including 95C, 95D, H1975 and A549, using RT-PCR. In accordance with previous studies, all 4 cell lines constitutively expressed CXCR4 mRNA, whereas CXCL12 mRNA expression was not observed (Fig. 1A). Following this, total cell lysates of these cell lines were prepared and examined for CXCL12 and CXCR4 protein expression by western blot analysis using CXCL12- and CXCR4-specific antibodies. As demonstrated in Fig. 1B, all 4 cell lines constitutively expressed CXCR4 protein but not its cognate ligand CXCL12.

Construction of functional CXCL12/CXCR4 autocrine loop in NSCLC cell line A549. The human NSCLC cell line A549 was transfected with pIRES2-ZsGreen1 plasmid encoding human CXCL12 or empty vector pIRES2-ZsGreen1 as a control, followed by selection with G418 to yield a stable CXCL12-expressing cell line (A549-CXCL12) and a stable control plasmid transfectant (A549-ZsGreen1). Using western blot analysis, CXCL12 protein expression was observed in A549-CXCL12 cells, but not in A549-ZsGreen1 or wild-type A549 cells (Fig. 2A). Furthermore, p-ERK1/2 was detected in A549-CXCL12 cells but not A549-ZsGreen1 cells (Fig. 2B), indicating autonomous ERK1/2 activation through CXCL12 binding to its cognate receptor CXCR4 in A549-CXCL12 cells. Taken together, these data indicated that a functional CXCL12/CXCR4 autocrine loop was successfully constructed in the A549-CXCL12 cell line.

CXCL12/CXCR4 autocrine loop promotes cell migration, invasiveness and proliferation. To investigate the influence of the CXCL12/CXCR4 autocrine loop on the malignant phenotype of NSCLC cells, we measured the migration, invasiveness and proliferation of A549-CXCL12, A549-ZsGreen1 and wild-type A549 cells. In wound migration analyses, A549-CXCL12 cells demonstrated significantly increased mobility compared with A549-ZsGreen1 and wild-type A549 cells $(\mathrm{P}<0.01$; Fig. $3 \mathrm{~A}$ and $\mathrm{C})$. The invasive potential of A549-CXCL12, A549-ZsGreen1 and wild-type A549 cells was determined using a Matrigel chamber assay. The number of invading A549-CXCL12 cells was significantly higher than (triple) that of A549-ZsGreen1 or wild-type A549 cells $(\mathrm{P}<0.01$; Fig. 3B and D). The MTT (3-(4,5-dimethylthi-

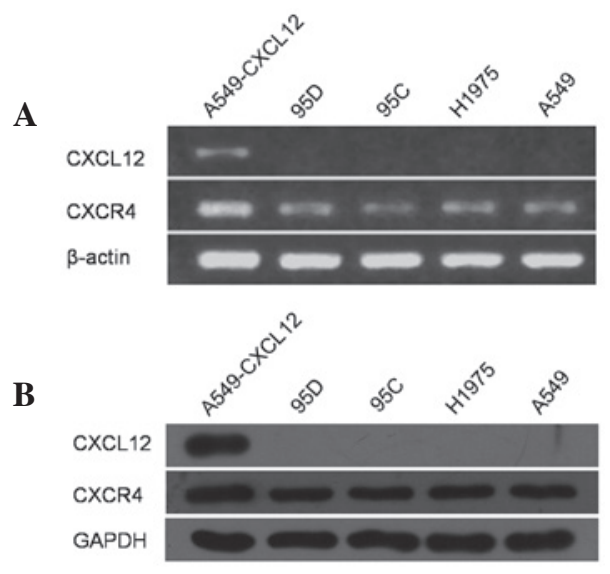

Figure 1. The expression patterns of CXCL12 and CXCR4 in non-small cell lung cancer (NSCLC) cell lines. Total RNA and proteins were extracted from 5 cell lines and subjected to RT-PCR and western blot analysis, respectively, for CXCL12 and CXCR4. Both mRNA (A) and protein (B) expression of CXCR4 but not CXCL12 were observed consistently in 95D, 95C, H1975, and A549 cell lines. The stable CXCL12 transfectant (A549-CXCL12) was used as a CXCL12 positive-control cell line. Data are representative of 3 independent analyses.
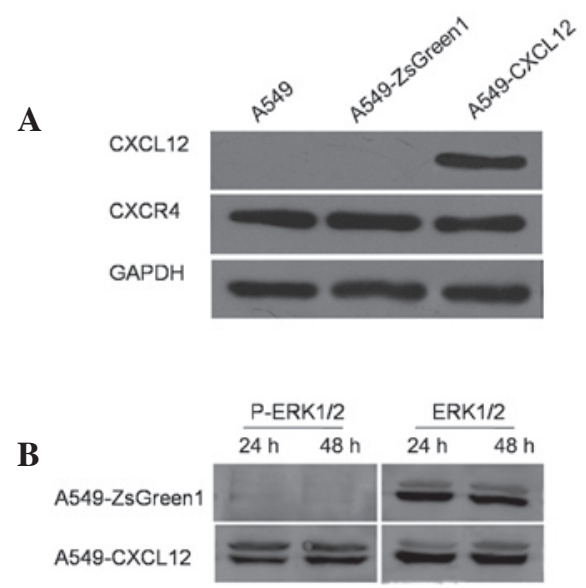

Figure 2. Functional expression of the CXCL12/CXCR4 autocrine loop in A549-CXCL12 cells. A549 cells were stably transfected with pIRES2-ZsGreen1-CXCL12 or empty vector pIRES2-ZsGreen1 to generate the stable transfectants A549-CXCL12 and A549-ZsGreen1. The cells were logarithmically grown, harvested and subjected to western blot analysis to detect CXCL12 and CXCR4 expression (A). Serum-starved cells were harvested at the indicated time points and subjected to western blot analysis for ERK1/2, using antibodies that recognized phosphorylated or total ERK1/2 (B). The results indicated that the functional CXCL12/CXCR4 autocrine loop was constructed successfully in A549-CXCL12 cells. Data are representative of 3 independent analyses.

azol-2-yl)-2,5-diphenyltetrazolium bromide) cell proliferation assay was performed to measure cell proliferation rate. As shown by the proliferation curve (Fig. 3E), the cell proliferation rate of A549-CXCL12 cells was markedly higher than that of A549-ZsGreen1 or wild-type A549 cells at 24 and $48 \mathrm{~h}$ $(\mathrm{P}<0.01)$, indicating that the CXCL12/CXCR4 autocrine loop induced increased cellular proliferation.

CXCL12/CXCR4 autocrine loop upregulates MMP-2 and MMP-9 expression. Tumor cell invasion and metastasis 
A
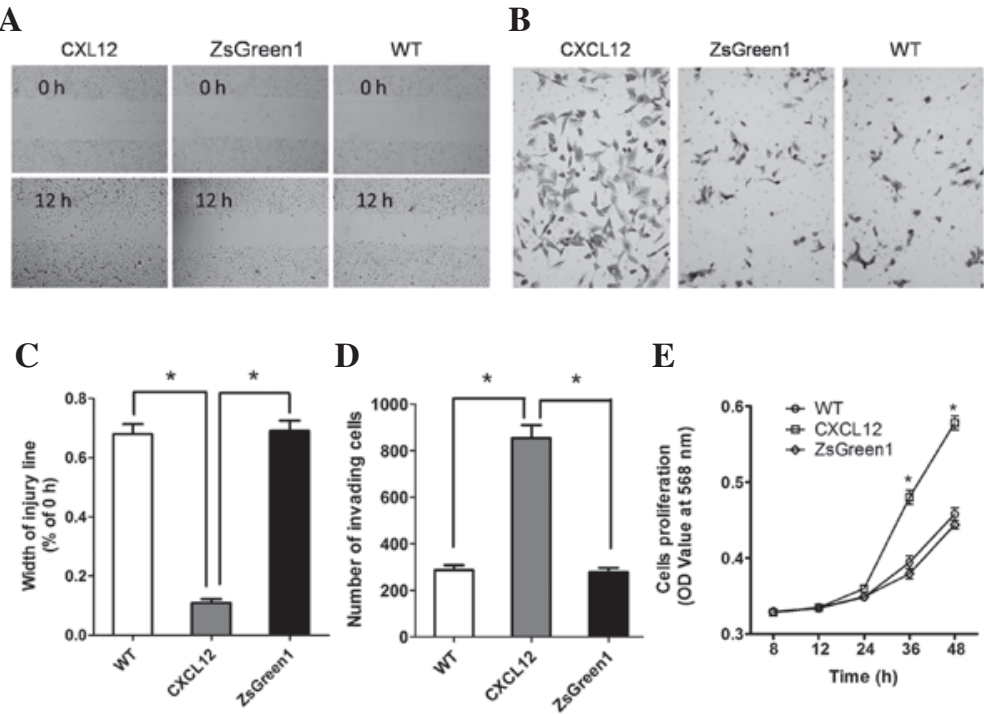

$\mathbf{E}$

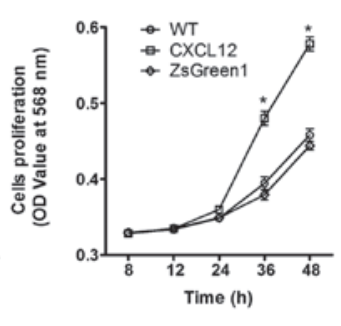

Figure 3. The CXCL12/CXCR4 autocrine loop increases non-small cell lung cancer (NSCLC) cell motility, invasion and proliferation. An injury line was created on confluent monolayers of A549-CXCL12 (CXCL12), A549-ZsGreen1 (ZsGreen1) and A549 (WT) cells. Cell motility was examined with a light microscope (x100) at the same location $12 \mathrm{~h}$ later (A) and the wounding width was quantified (C). Cells were seeded onto a Matrigel invasion chamber. Samples were then incubated for $18 \mathrm{~h}$ in serum-free RPMI-1640 medium. After staining, the cells that invaded through the Matrigel-coated membrane were counted in 9 fields under a light microscope (x400) (B and D). The growth curve was constructed by plotting absorbance against time using the MTT method (E). Values in $\mathrm{B}, \mathrm{D}$ and $\mathrm{E}$ represent the mean $\pm \mathrm{SD}$ of 3 independent experiments. ${ }^{*}$ indicates a statistically significant difference between A549-CXCL12 and control cells $(\mathrm{P}<0.01)$.
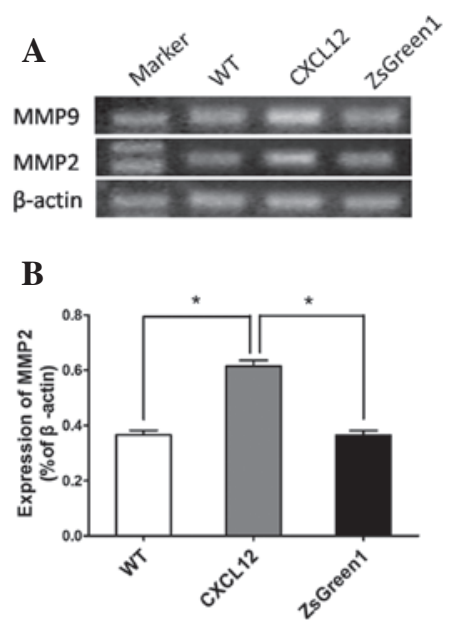

C

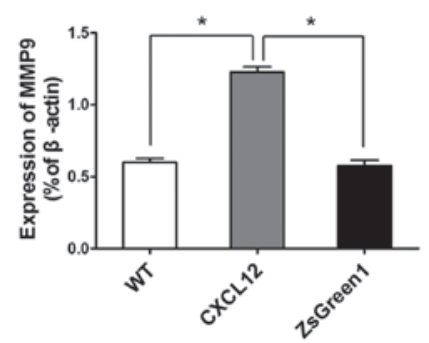

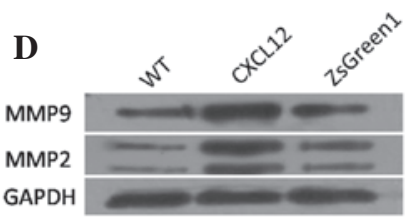

E

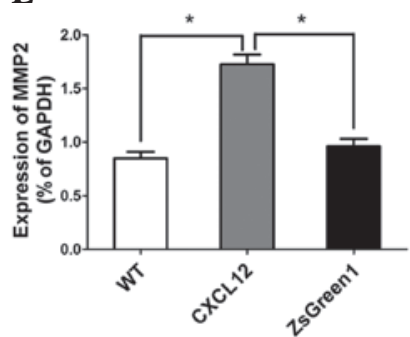

$\mathbf{F}$

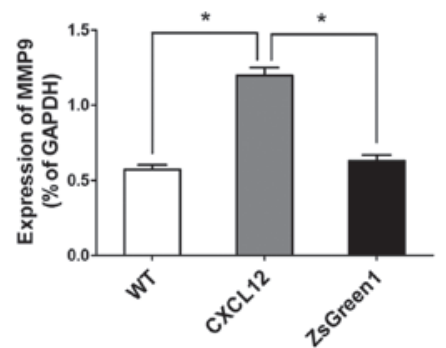

Figure 4. The CXCL12/CXCR4 autocrine loop upregulates MMP-2 and MMP-9 expression. Total RNA and proteins were extracted from the 3 cell lines and subjected to RT-PCR (A, B and C) or western blot analysis (D, E and F) for MMP-2 and MMP-9. Values represent the mean \pm SD of 3 independent experiments performed in triplicate. ${ }^{*}$ indicates a statistically significant difference between A549-CXCL12 and control cells $(\mathrm{P}<0.01)$

involve ECM degradation and remodeling in addition to cell locomotion. MMP-2 and MMP-9 are capable of degrading several ECM proteins, including type IV and V collagens, laminin and elastin. To investigate whether the CXCL12/
CXCR4 autocrine loop influences MMP-2 and MMP-9 expression, we performed RT-PCR to detect mRNA expression in A549-CXCL12, A549-ZsGreen1 and wild-type A549 cells. Markedly increased MMP-2 and MMP-9 expression 
was observed in A549-CXCL12 cells compared with control cells (Fig. 4A-C). Western blot analysis also revealed significantly higher expression levels of MMP-2 and MMP-9 in A549-CXCL12 cells.

\section{Discussion}

Primary non-small cell lung cancer is the leading cause of cancer mortality worldwide. Most patients present with locally advanced (37\%) or metastatic (38\%) disease at the time of diagnosis (21). As with most cancers, early-stage NSCLC is often be controlled with locally directed therapy including radiation and surgery; it is the development of metastatic disease that leads to the high mortality rate of NSCLC. Therefore, possible mechanisms of metastasis, as well as the early detection and screening of lung cancer, have been the subject of growing interest.

Tumor metastasis is an organized process that occurs in a stepwise fashion: i) uncontrolled proliferation and local invasion; ii) intravasation into the vascular system and survival in the circulation; iii) escape of cancer cells from the lumina of blood vessels into the parenchyma of distant tissues (extravasation), and iv) the formation of secondary tumors (colonization) (22-24). Tumor metastasis is also a non-random, highly organ-specific pathophysiological process $(23,25,26)$. A growing body of evidence has indicated that the chemokine CXCL12 and its cognate receptor CXCR4 are critical in this process $(6,10,27-30)$. These studies have led to the current CXCL12/CXCR4 'endocrine axis' model; CXCR4 expression by metastatic cells enables these cells to navigate towards organs abundantly expressing CXCL12. In the case of NSCLC, the role of the CXCL12/CXCR4 endocrine axis has been well established (10), but the precise effect of the CXCL12/CXCR4 autocrine loop on NSCLC cells has not yet been demonstrated. Several studies have been conducted to evaluate the functionality of the CXCL12/CXCR4 autocrine loop in human mammary carcinoma $(11,12)$, oral squamous cell carcinoma (31) and colorectal carcinoma cells (32). However, the evidence arising from these studies has been controversial and even contradictory.

In this study, CXCR4 was constitutively expressed in all 4 human NSCLC cell lines evaluated, but $\mathrm{CXC12}$ was not observed consistently. To investigate the effect of the CXCL12/ CXCR4 autocrine loop on the NSCLC cells, the full-length CXCL12 gene was transfected into human NSCLC cell line A549 to generate the stable CXCL12-expressing transfectant, A549-CXCL12. A549-CXCL12 cells overexpressed CXCL12 and autonomous ERK1/2 activation was also observed, indicating that this transfectant had acquired a functional CXCL12/CXCR4 autocrine loop. The ability to migrate was evaluated first, as this is a significant characteristic of the aggressive phenotype of malignant tumor cells. Our results demonstrated that the CXCL12/CXCR4 autocrine loop signaling pathway significantly enhanced A549-CXCL12 cell migration compared with control A549-ZsGreen1 and wild-type A549 cells $(\mathrm{P}<0.01)$. These results are consistent with those revealed by Kang et al (11) and Uchida et al (31), who performed similar experiments using human mammary carcinoma cells and oral squamous cell carcinoma cells, respectively. Metastasis fundamentally involves the move- ment of cells from one site to another. These results indicated that the CXCL12/CXCR4 autocrine loop may promote the metastatic potential of NSCLC cells.

The most fundamental trait of malignant tumor cells is their ability to sustain chronic proliferation. Thus, another significant characteristic of the aggressive phenotype of malignant tumor cells is uncontrolled cellular proliferation. As a primary tumor grows, its blood supply cannot support its metabolic needs; lack of oxygen causes tumor cells to move away from the site of hypoxia and spread to new locations through activation of genes such as $c$-Met and CXCR4 (33). Our results revealed that the CXCL12/ CXCR4 autocrine loop induced a significant increase in A549-CXCL12 cell proliferation compared with the vector control cell line A549-ZsGreen1 and wild-type parent cell line A549, indicating that the CXCL12/CXCR4 autocrine loop may play a critical role in tumor growth so as to facilitate tumor metastasis. These results are in accordance with studies demonstrating that the CXCL12/CXCR4 autocrine loop increased mammary carcinoma cell proliferation (13). However, they are in disagreement with previous results by Wendt et al (33), who revealed increased apoptosis in forced CXCL12-expressing colorectal cancer cells compared to control eGFP clones. These data highlight differences in the pathophysiological impact of the CXCL12/CXCR4 autocrine loop among different types of cancer cells.

In the present study, the CXCL12/CXCR4 autocrine loop also markedly enhanced the invasiveness of NSCLC cells. Kang et al and Uchida et al $(11,31)$ reported that the CXCL12/CXCR4 autocrine loop significantly increased breast cancer and oral squamous cell carcinoma cell invasiveness, respectively. By contrast, Wendt et al reported decreased invasiveness and metastasis in breast cancer cells and colorectal cancer cells in vivo $(12,32)$, suggesting that the role of the CXCL12/CXCR4 autocrine loop in modulating the capacity for invasiveness may depend on the type of cancer. Enzymatic degradation of ECM and basement membranes is a key step in cancer invasion and metastasis. In human lung cancer, MMP-2 and MMP-9 have been demonstrated to be correlated with malignancy grade and metastasis $(13,34)$. Therefore, we hypothesized that the CXCL12/CXCR4 autocrine loop may stimulate MMP-2 and MMP-9 production to degrade the ECM and basement membranes. Both mRNA and protein expression of MMP-2 and MMP-9 were markedly increased in A549-CXCL12 cells compared with controls, further supporting the notion that the CXCL12/ CXCR4 autocrine loop indirectly enhanced the capacity for NSCLC cell invasiveness.

Overall, these results indicate that the CXCL12/CXCR4 autocrine loop increases the metastatic potential of NSCLC cells, confirming that the CXCL12/CXCR4 autocrine loop may contribute to the motility, growth and invasiveness of NSCLC. To our knowledge, this in vitro study is the first to reveal that the CXCL12/CXCR4 autocrine loop increases the metastatic potential of NSCLC. Furthermore, our findings suggest that targeted therapies against CXCR4 (35) should consider the CXCL12 expression status of the NSCLC to be treated, since tumors with autocrine overexpression of CXCL12 may be more sensitive to CXCR4 antagonists that compete with CXCL12 for receptor binding and more suitable 
for the application of chemokine-based anti-cancer therapies. Differences in the impact of the CXCL12/CXCR4 autocrine loop on cancer cells may depend on the type of cancer, and this possibility requires further investigation.

\section{References}

1. Vindrieux D, Escobar P and Lazennec G: Emerging roles of chemokines in prostate cancer. Endocr Relat Cancer 16: 663-673, 2009.

2. Slettenaar VI and Wilson JL: The chemokine network: a target in cancer biology? Adv Drug Deliv Rev 58: 962-974, 2006.

3. Balkwill F: Cancer and the chemokine network. Nat Rev Cancer 4: 540-550, 2004.

4. Ben-Baruch A: The multifaceted roles of chemokines in malignancy. Cancer Metastasis Rev 25: 357-371, 2006.

5. Keeley EC, Mehrad B and Strieter RM: CXC chemokines in cancer angiogenesis and metastases. Adv Cancer Res 106: 91-111, 2010

6. Müller A, Homey B, Soto H, et al: Involvement of chemokine receptors in breast cancer metastasis. Nature 410: 50-56, 2001.

7. Rubie C, Frick VO, Ghadjar P, et al: CXC receptor- 4 mRNA silencing abrogates CXCL12-induced migration of colorectal cancer cells. J Transl Med 9: 22, 2011.

8. Liang JJ, Zhu S, Bruggeman R, et al: High levels of expression of human stromal cell-derived factor-1 are associated with worse prognosis in patients with stage II pancreatic ductal adenocarcinoma. Cancer Epidemiol Biomarkers Prev 19: 2598-2604, 2010.

9. Castellone MD, Guarino V, De Falco V, et al: Functional expression of the CXCR4 chemokine receptor is induced by RET/PTC oncogenes and is a common event in human papillary thyroid carcinomas. Oncogene 23: 5958-5967, 2004.

10. Phillips RJ, Burdick MD, Lutz M, Belperio JA, Keane MP and Strieter RM: The stromal derived factor-1/CXCL12-CXC chemokine receptor 4 biological axis in non-small cell lung cancer metastases. Am J Respir Crit Care Med 167: 1676-1686, 2003.

11. Kang H, Watkins G, Parr C, Douglas-Jones A, Mansel RE and Jiang WG: Stromal cell derived factor-1: its influence on invasiveness and migration of breast cancer cells in vitro, and its association with prognosis and survival in human breast cancer. Breast Cancer Res 7: R402-410, 2005

12. Wendt MK, Cooper AN and Dwinell MB: Epigenetic silencing of CXCL12 increases the metastatic potential of mammary carcinoma cells. Oncogene 27: 1461-1471, 2008.

13. Deryugina EI and Quigley JP: Matrix metalloproteinases and tumor metastasis. Cancer Metastasis Rev 25: 9-34, 2006.

14. Ii M, Yamamoto H, Adachi Y, Maruyama Y and Shinomura Y: Role of matrix metalloproteinase-7 (matrilysin) in human cancer invasion, apoptosis, growth, and angiogenesis. Exp Biol Med (Maywood) 231: 20-27, 2006.

15. Tang $\mathrm{CH}$, Tan TW, Fu WM and Yang RS: Involvement of matrix metalloproteinase-9 in stromal cell-derived factor-1/CXCR4 pathway of lung cancer metastasis. Carcinogenesis 29: 35-43, 2008.

16. Chakrabarti S and Patel KD: Matrix metalloproteinase-2 (MMP-2) and MMP-9 in pulmonary pathology. Exp Lung Res 31: 599-621, 2005.
17. Iijima T, Minami Y, Nakamura N, et al: MMP-2 activation and stepwise progression of pulmonary adenocarcinoma: analysis of MMP-2 and MMP-9 with gelatin zymography. Pathol Int 54: 295-301, 2004

18. Okada N, Ishida H, Murata N, Hashimoto D, Seyama Y and Kubota S: Matrix metalloproteinase-2 and -9 in bile as a marker of liver metastasis in colorectal cancer. Biochem Biophys Res Commun 288: 212-216, 2001

19. Rao JS, Gondi C, Chetty C, Chittivelu S, Joseph PA and Lakka SS: Inhibition of invasion, angiogenesis, tumor growth, and metastasis by adenovirus-mediated transfer of antisense UPAR and MMP-9 in non-small cell lung cancer cells. Mol Cancer Ther 4: 1399-1408, 2005.

20. Su L, Zhang J, Xu H, et al: Differential expression of CXCR4 is associated with the metastatic potential of human non-small cell lung cancer cells. Clin Cancer Res 11: 8273-8280, 2005.

21. Jemal A, Murray T, Ward E, et al: Cancer statistics, 2005. CA Cancer J Clin 55: 10-30, 2005.

22. Talmadge JE and Fidler IJ: AACR centennial series: the biology of cancer metastasis: historical perspective. Cancer Res 70: 5649-5669, 2010

23. Fidler IJ: The pathogenesis of cancer metastasis: the 'seed and soil' hypothesis revisited. Nat Rev Cancer 3: 453-458, 2003.

24. Geiger TR and Peeper DS: Metastasis mechanisms. Biochim Biophys Acta 1796: 293-308, 2009.

25. Hynes RO: Metastatic potential: generic predisposition of the primary tumor or rare, metastatic variants-or both? Cell 113: 821-823, 2003

26. Liotta LA: An attractive force in metastasis. Nature 410: 24-25, 2001.

27. Liang Z, Yoon Y, Votaw J, Goodman MM, Williams L and Shim $\mathrm{H}$ : Silencing of CXCR4 blocks breast cancer metastasis. Cancer Res 65: 967-971, 2005.

28. do Carmo A, Patricio I, Cruz MT, Carvalheiro H, Oliveira CR and Lopes MC: CXCL12/CXCR4 promotes motility and proliferation of glioma cells. Cancer Biol Ther 9: 56-65, 2010.

29. Zlotnik A: Involvement of chemokine receptors in organ-specific metastasis. Contrib Microbiol 13: 191-199, 2006.

30. Wagner PL, Hyjek E, Vazquez MF, et al: CXCL12 and CXCR4 in adenocarcinoma of the lung: association with metastasis and survival. J Thorac Cardiovasc Surg 137: 615-621, 2009.

31. Uchida D, Onoue T, Tomizuka Y, et al: Involvement of an autocrine stromal cell derived factor-1/CXCR4 system on the distant metastasis of human oral squamous cell carcinoma. Mol Cancer Res 5: 685-694, 2007.

32. Wendt MK, Johanesen PA, Kang-Decker N, Binion DG, Shah V and Dwinell MB: Silencing of epithelial CXCL12 expression by DNA hypermethylation promotes colonic carcinoma metastasis. Oncogene 25: 4986-4997, 2006.

33. Staller P, Sulitkova J, Lisztwan J, Moch H, Oakeley EJ and Krek W: Chemokine receptor CXCR4 downregulated by von Hippel-Lindau tumour suppressor pVHL. Nature 425: 307-311, 2003.

34. Hsu CP, Shen GH and Ko JL: Matrix metalloproteinase-13 expression is associated with bone marrow microinvolvement and prognosis in non-small cell lung cancer. Lung Cancer 52: 349-357, 2006.

35. Gangadhar T, Nandi S and Salgia R: The role of chemokine receptor CXCR4 in lung cancer. Cancer Biol Ther 9: 409-416, 2010 . 